Original Article

\title{
LC-MS/MS STUDY OF THE TRACE LEVEL IMPURITIES OF IRBESARTAN AN ANGIOTENSIN II RECEPTOR ANTAGONIST MOLECULE TO ITS ORIGIN THROUGH nMS ${ }^{2}$ TECHNIQUE
}

\author{
S. SARAVANAN ${ }^{1,2}$, A. TAMINUM ANSARI²
}

${ }^{1}$ Research and Development Centre, Bharathiar University, Coimbatore, India, ${ }^{2}$ Indian Institute of Chromatography and Mass Spectrometry, Spinco Biotech, Spinco Towers, Chennai, India, ${ }^{3}$ Muthurangam Govt Arts College, Vellore

Email: sar_ash_25@yahoo.co.in

Received: 09 Aug 2018 Revised and Accepted: 28 Nov 2018

\begin{abstract}
Objective: The mass characterization of five trace level related impurities of Irbesartan was performed through the "nMS2" technique of triple quadrupole mass spectrometer and also to Correlated to the impurity origin.

Methods: A simple \& effective patented process was applied to get the impurity profile, and mass characterization was performed through the "nMS2" technique of triple quadrupole mass spectrometer analyser.

Results: The simple production scan in differential collision energies is coined as "nMS2" technique of the triple quadrupole analyzer. The molecular ion fragmentation occurs with multiple collision energies and provides meaningful MSMS fragments for characterizing five trace level impurities less than $0.5 \%$ of angiotensin II receptor antagonist-Irbesartan. The origin of the impurity formation in the synthetic process was successfully related to the Spiro ketones.
\end{abstract}

Conclusion: The results obtained in this research clearly indicates the approach of "nMS" technique was very useful in the identification and structural prediction of trace level related impurities of Irbesrtan.

Keywords: nMS²technique, Irbesartan, LCMSMS of Irbesartan, Impurity Characterization

(C) 2019 The Authors. Published by Innovare Academic Sciences Pvt Ltd. This is an open-access article under the CC BY license (http://creativecommons.org/licenses/by/4.0/) DOI: http://dx.doi.org/10.22159/ijpps.2019v11i1.29037

\section{INTRODUCTION}

Safety and efficacy of pharmaceutical substances are the two fundamental issues in drug therapy. The safety of a drug is determined by its pharmacological or toxicological profile as well as the adverse effects caused by the impurities in bulk and dosage forms. The drug should be safe, i.e. it should have an acceptably low risk of adverse effects with doses of drug which provide the desired therapeutic effects. The impurities in drugs often possess unwanted pharmacological or toxicological effects by which any benefit from their administration may be outweighed. Thus the quality of the drug is directly related to safety. The quality and safety of a drug is generally assured by monitoring it using suitable analytical techniques and controlling the Impurities effectively. Thus, the analytical activities concerning impurities in drugs are among the most important aspect in the modern pharmaceutical analysis [1-5]. The International Conference on Harmonization (ICH) guidelines defines the impurity as "Any component of the medicinal product which is not the chemical entity defined as the active substance or an excipient in the product". The impurity profiling is hence considered to be the analytical activities with the aim of detecting, identifying or elucidating the structure and quantitatively determining impurities in bulk drugs and pharmaceutical formulations [6-10].

The impurities were broadly classified as related and non-related impurities. In pharmaceuticals the manufacturing facilities follows stringent manufacturing practices, thus the possibility for the nonrelated unknown impurity formation will be very rare considerably difficult to identify and characterize this totally unknown impurity. Development of a cost-effective process is always desirable for sustained commercialization of any drug substances. Therefore, continuous innovations are constantly employed by process chemist to implement cost-effective synthesis scheme in scale-up operations and hence successful commercialization of the drug substances. This kind of effort requires the use of commercially available conventional raw materials along with plant-based solvents for the process, workup and purification of the final product ensuring the quality of the product. This report demonstrates how the MSMS architecture of LCMS-8040 acts as an "enabler" in identifying process-related impurities appropriately to ensure the quality of the drug substance Irbesartan. Irbesartan is the angiotensin II receptor antagonists intended for the treatment of hypertension and also for the delay progression of diabetic nephropathy. It is often formulated with a lesser dosage level of thiazide diuretic The salient features of LCMS 8040 like Synchronized Survey Scan (SSS) provides the MS and MSMS data simultaneously in a single run and paves the way for rapid identification of impurities. Further, the simultaneous MS analysis in both positive and negative modes, MSMS analysis with different collision energies and MSMS analysis with neutral loss function have enabled us to characterize the impurities of Irbesartan.

\section{MATERIALS AND METHODS}

Chemicals and reagents

The synthetic chemicals are LR grade for step 1 and step2 of synthetic reaction scheme. AR grade reagents are used for step3. Cyano methyl biphenyl and spiroketone is procured from LobaChemi along with scheme reagents. Analytical grade reagents and LCMS grade solvents is used for the LCMS and LCMSMS analysis.

\section{Instrumentation}

An UFMS consisting of the Shimadzu UHPLC NEXERA as a frontend for the Shimadzu LCMS 8040 triple quadrupole mass spectrometer. A reverse phase Shimadzu shimpak XR-ODS (50 X3.0 mm, $2.2 \mu \mathrm{m}$ ) column was used for the chromatographic separation. The chromatographic data is recorded with Shimadzu Lab solution software. The LCMS and LCMSMS were performed with the FCV12 divert valve arrangement. ESI was used as the ionization source for the mass analysis in both positive and negative polarity.

\section{Chromatographic and mass spectrometric conditions}

The UHPLC separation was carried out with Shimadzu shimpak XRODS (50 X3.0 mm, $2.2 \mu \mathrm{m}$ ) column using $0.1 \%$ fomic acid in water at 
$3.4 \mathrm{pH}$ and acetonitrile as mobile phase in a gradient mode. The time $/ \% \mathrm{~B}$ concentration of the gradient programme is $0.01 / 33$, $2.00 / 33,10.00 / 60,13.00 / 60,15.00 / 33,17.00 / 33$. The flow rate was $0.3 \mathrm{ml} / \mathrm{min}$, and the injection volume is $10 \mu \mathrm{L}$. LC-MS/MS was carried out with Electrospray ionization (ESI) in positive and negative mode of detection. Nitrogen was used as a nebulizer gas. Collision-induced dissociation was achieved by argon as collision gas. The operating conditions for MS scan were: DL temperature $250{ }^{\circ} \mathrm{C}$; nebulizer gas, $3.0 \mathrm{~L} / \mathrm{min}$; dry gas, $15.0 \mathrm{~L} / \mathrm{min}$; ESI voltage $4.5 \mathrm{kV}$; heat block, $350{ }^{\circ} \mathrm{C}$ and the sacn range, 150 to $2000 \mathrm{amu}$. The selected quazi molecular ion was subjected to the LCMSMS analysis in 11 different collision energies (CE $=5 \mathrm{v}$ to $60 \mathrm{v}$ in increment of 5 ).

\section{DISCUSSION}

Irbesartan was in-house synthesized at Indian Institute of chromatography and Mass Spectrometry (IICMS), Chennai, India for academic research. A three-stage synthetic root is adapted as per EP1918288A1 [11]. The synthetic scheme is shown in fig. 1.

The step 1(S-1) corresponds to alkyl bromination followed by spiroketone substitution in step2 (S-2) and cyclization with a reduction to form tetrazole ring in step3(S-3). The purity of the compound was found to be $99.21 \%$ (Area normalization method) with five trace level impurities.

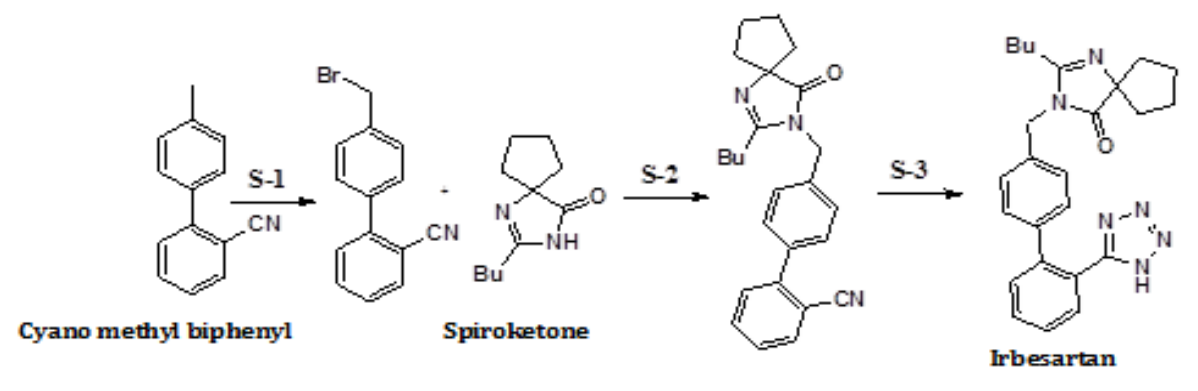

Fig. 1: Synthetic scheme for Irbesartan [11]

Though a three-step process is used to synthesize Irbesartan, the HPLC analysis shows the presence of five impurities, whose concentration varies from $0.04 \%$ to $0.32 \%$ Area (table 1). Fig. 2 shows the HPLC chromatogram of Irbesartan and its five trace level impurities. The LCMS analysis was performed for the above five impurities.

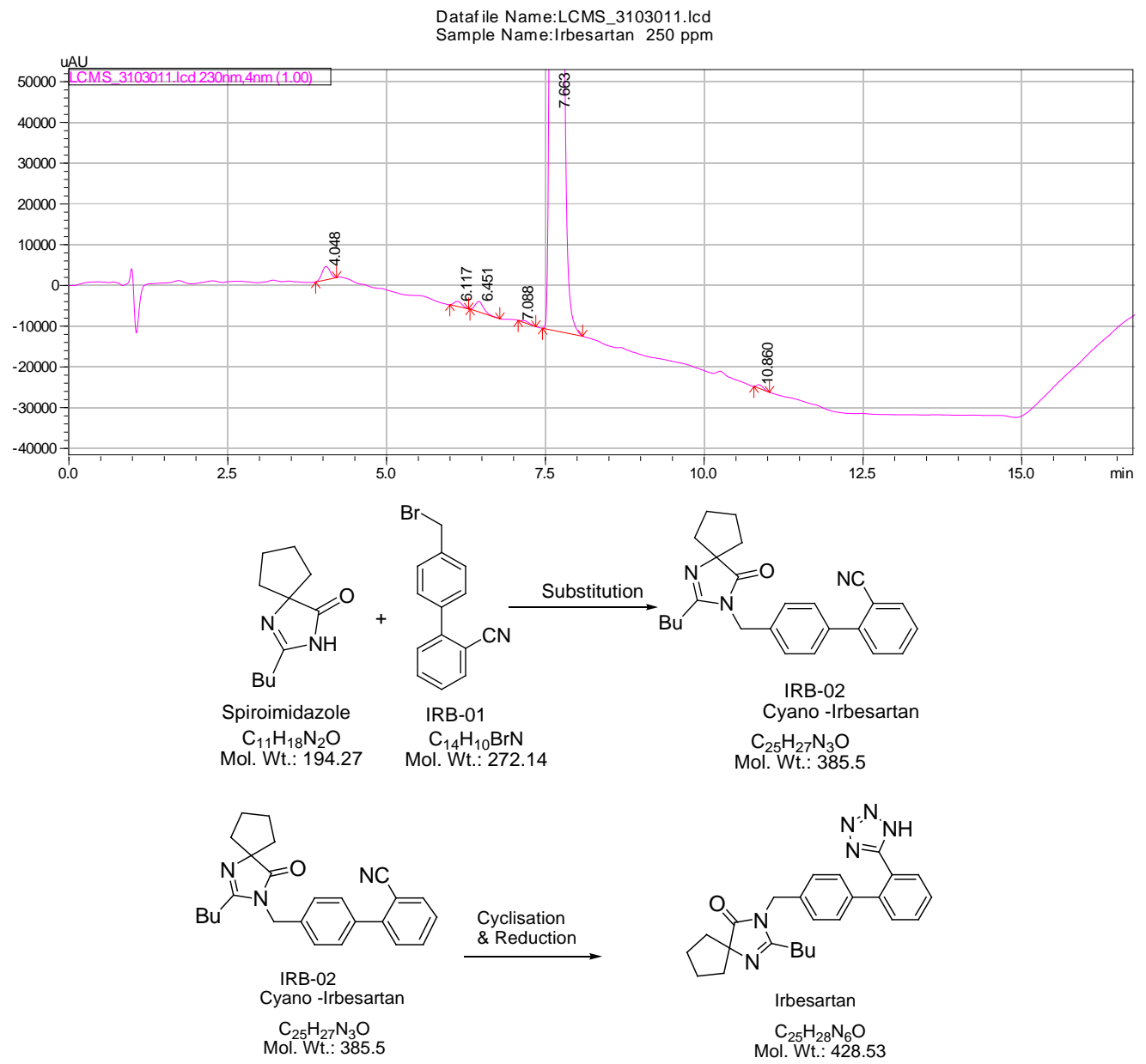

Fig. 2: HPLC chromatogram of Irbesartan and its trace level impurities 
Table 1: The Retention time and area percentage of Irbesartan and impurities

\begin{tabular}{lll}
\hline Impurities & Retention time (min) & Level of impurities in UV detection (\%Area) \\
\hline Impurity-1 & 4.05 & 0.32 \\
Impurity-2 & 6.12 & 0.11 \\
Impurity-3 & 6.45 & 0.26 \\
Impurity-4 & 7.09 & 0.04 \\
Impurity-5 & 10.86 & 0.07 \\
\hline
\end{tabular}

The trace level impurity area is shown in table 1

The impurity variance is between $0.04 \% \mathrm{a} / \mathrm{a}$ to $0.32 \% \mathrm{a} / \mathrm{a}$. Thus it is less than $0.5 \% \mathrm{a} / \mathrm{a}$ in the analyzed chromatographic conditions [1214]. On comparing the polarity in the chromatographic impurity elution order, it was observed that four impurities are polar with respect to irbesartan and the one with later elution is relatively nonpolar to irbesartan. This also infers that the structure of these impurities has functional group variances or aliphatic chain increments/decrements reflecting to polarity.
The mass spectrometric analysis in impurity characterization is the first approach for the possible clue for the formation of impurity in the reaction process of the intended product. The Irbesartan and its impurities were mass analyzed. The molecular ion of Irbesartan has been observed as $\mathrm{m} / \mathrm{z} 429[\mathrm{M}+1]$ in ESI positive mode, confirmed in the simultaneous ESI negative mode analysis as m/z 427 [M-1]. Similar to irbesartan, the molecular mass of the impurities was also confirmed by the simultaneous positive $[\mathrm{M}+1]$ ions and negative [M-1] ions and tabulated in table 2 .

Table 2: Molecular mass of Irbesartan and impurities

\begin{tabular}{llll}
\hline Name & Molecular ion & Molecular mass (Da) \\
\cline { 2 - 3 } & Positive & Negative & 372 \\
\hline Impurity 1 & 373 & 371 & 414 \\
Impurity 2 & 415 & 413 & 446 \\
Impurity 3 & 447 & 445 & 426 \\
Impurity 4 & 427 & 425 & 442 \\
Impurity 5 & 443 & 441 & \\
\hline
\end{tabular}

The impurities are correlated to Irbesartan and found all the impurities are behaving similar in mass spectrometry with ionization both in positive and negative ionization.

\section{The LCMS/MS-"nMS" approach}

The sanctity of an LCMS analysis remains in the novel approach of its intended application. The stringent specification of international regulatory bodies has made it necessary to characterize the impurities in trace level - $(0.1 \%)$. This is to minimize the adverse biological effects of the unknown impurities and also to improve the potency of the active component. Characterization of a compound and its trace level impurities requires MS and MSMS analysis. The segmental analysis was performed to obtain the above molecular masses for irbesartan. The MSMS analysis of irbesartan with nine different collision energies simultaneously in ESI positive ionization shows the characteristic fragmentation ions such as $\mathrm{m} / \mathrm{z} 429, \mathrm{~m} / \mathrm{z} 207, \mathrm{~m} / \mathrm{z} 195, \mathrm{~m} / \mathrm{z} 180$ and m/z 84 of irbeartanis shown in table 3 and the proposed fragmentation pathway is shown in fig. 3.

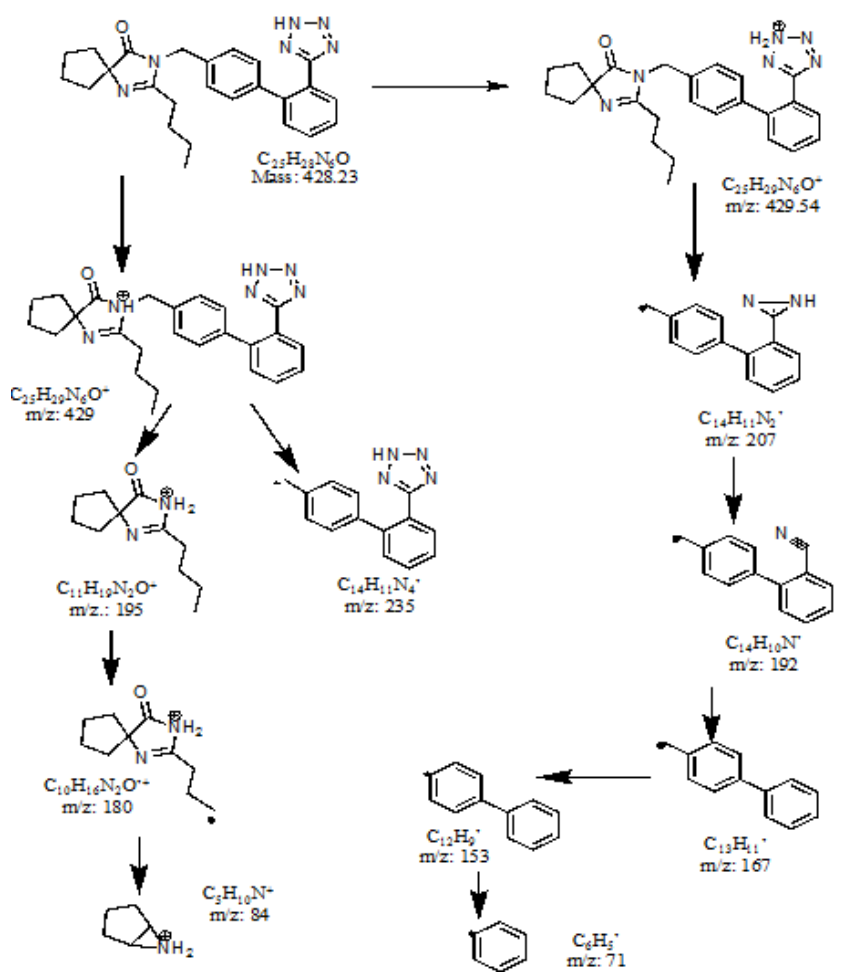

Fig. 3: The proposed fragmentation pathway of Irbesartan 
Table 3: Compiled mass fragments of Irbesartan

\begin{tabular}{|c|c|c|c|c|c|c|c|c|c|}
\hline $\mathrm{CE}$ & Irbe & & & & & & & & \\
\hline-5 & 429 & & & & & & & & \\
\hline-10 & 429 & 207 & & & & & & & \\
\hline-15 & 429 & 207 & 195 & & & & & & \\
\hline-20 & 429 & 207 & 195 & & & & & & \\
\hline-25 & & 207 & 195 & & & & & & \\
\hline-30 & & 207 & 195 & & 180 & & & & \\
\hline-35 & & 207 & 195 & 190,192 & 180 & & & 84 & \\
\hline-40 & & 207 & 195 & 190,192 & 180 & & 153 & 84 & \\
\hline-45 & & 207 & 195 & 190,192 & 180 & 165,167 & 153 & 84 & \\
\hline-50 & & 207 & 195 & 190,192 & 180 & 165,167 & 151 & 84 & 77 \\
\hline-60 & & 205 & & 190,192 & 180 & 165,167 & & 84 & 77 \\
\hline
\end{tabular}

The MS identified impurities are subjected to the MSMS analysis simultaneously at eleven different collision energies for the five impurities in both positive and negative ESI mode. The MSMS chromatogram and compiled mass fragments in positive mode is shown in fig. 4 and the MSMS chromatogram and compiled mass fragments in negative mode is shown in fig. 5.
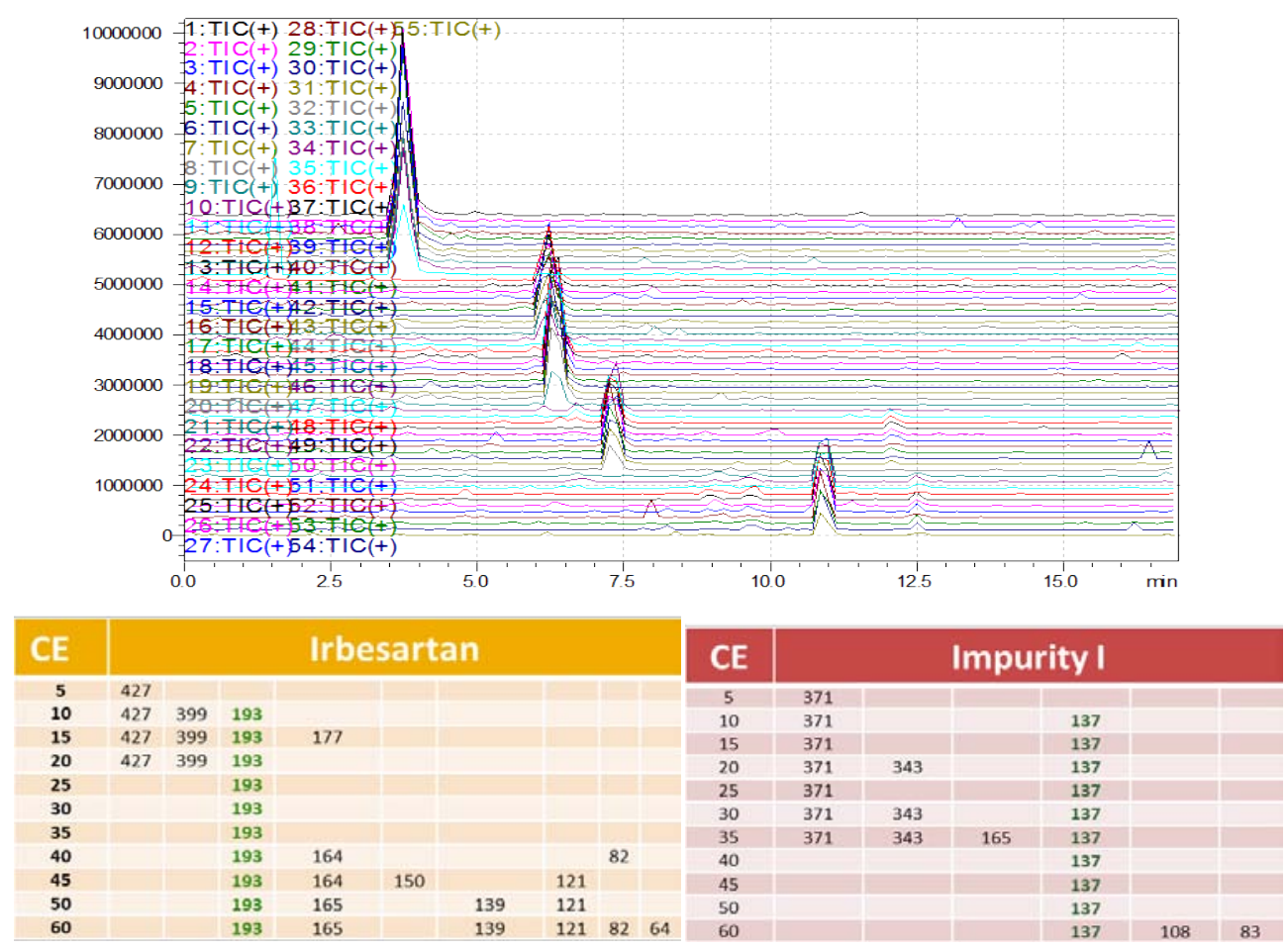

\begin{tabular}{|c|c|c|c|c|c|c|c|c|c|c|c|c|}
\hline CE & \multicolumn{4}{|c|}{ Impurity II } & CE & \multicolumn{7}{|c|}{ Impurity III } \\
\hline 5 & 413 & & & & 5 & 445 & & & & & & \\
\hline 10 & 413 & & & & 10 & 445 & & & & & & \\
\hline 15 & 413 & & & & 15 & 445 & & & & & & \\
\hline 20 & 413 & 385 & 179 & & 20 & 445 & 305 & & & & & \\
\hline 25 & 413 & 385 & 180 & & 25 & 445 & 305 & & & & & \\
\hline 30 & 413 & 385 & 179 & & 30 & 445 & 305 & 211 & 194 & 165 & 127 & 100 \\
\hline 35 & 413 & & 179 & & 35 & 445 & 305 & 212 & 194 & 165 & & \\
\hline 40 & 413 & & 179 & & 40 & 445 & 305 & 211 & 194 & 165 & 127 & \\
\hline 45 & & & 179 & & 45 & 445 & 305 & 211 & 194 & 165 & 127 & \\
\hline 50 & & & 179 & & so & 445 & 305 & 211 & 194 & 165 & 127 & \\
\hline 60 & & & 179 & 124 & 60 & & 305 & 211 & & 165 & 127 & 100 \\
\hline
\end{tabular}

\begin{tabular}{|c|c|c|}
\hline CE & \multicolumn{2}{|c|}{ Impurity IV } \\
\hline 5 & 425 & \\
\hline 10 & 425 & \\
\hline 15 & 425 & 191 \\
\hline 20 & 425 & 191 \\
\hline 25 & 425 & 191 \\
\hline 30 & 425 & 191 \\
\hline 35 & 425 & 191 \\
\hline 40 & 425 & 191 \\
\hline 50 & & 191 \\
\hline 60 & & 191 \\
\hline
\end{tabular}

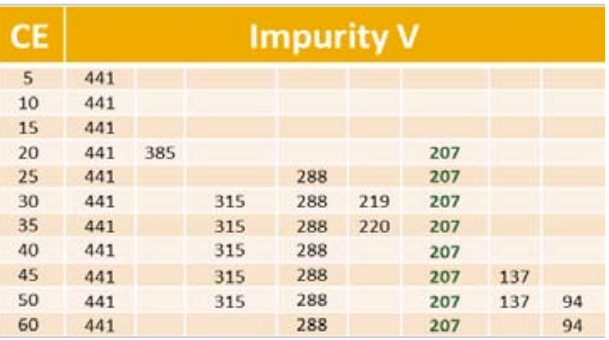

Fig. 4: The overlaid MSMS chromatogram of five impurities evaluated under 11 different collisions (55 data in 15 min) and compiled mass fragments in ESI Positive mode 

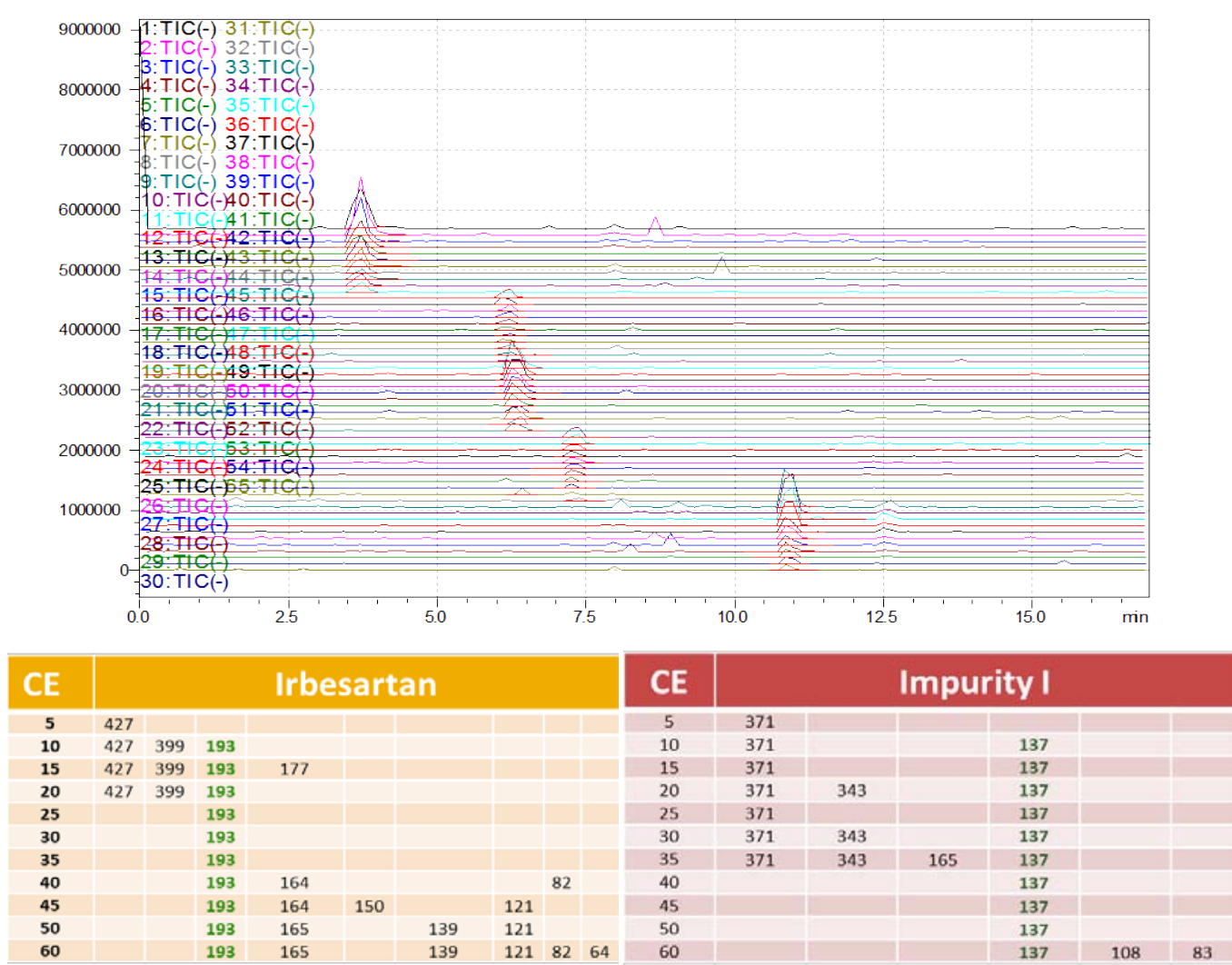

\begin{tabular}{|c|c|c|c|c|c|c|c|c|c|c|c|c|}
\hline CE & \multicolumn{4}{|c|}{ Impurity II } & CE & \multicolumn{7}{|c|}{ Impurity III } \\
\hline 5 & 413 & & & & 5 & 445 & & & & & & \\
\hline 10 & 413 & & & & 10 & 445 & & & & & & \\
\hline 15 & 413 & & & & 15 & 445 & & & & & & \\
\hline 20 & 413 & 385 & 179 & & 20 & 445 & 305 & & & & & \\
\hline 25 & 413 & 385 & 180 & & 25 & 445 & 305 & & & & & \\
\hline 30 & 413 & 385 & 179 & & 30 & 445 & 305 & 211 & 194 & 165 & 127 & 100 \\
\hline 35 & 413 & & 179 & & 35 & 445 & 305 & 212 & 194 & 165 & & \\
\hline 40 & 413 & & 179 & & 40 & 445 & 305 & 211 & 194 & 165 & 127 & \\
\hline 45 & & & 179 & & 45 & 445 & 305 & 211 & 194 & 165 & 127 & \\
\hline 50 & & & 179 & & 50 & 445 & 305 & 211 & 194 & 165 & 127 & \\
\hline 60 & & & 179 & 124 & 60 & & 305 & 211 & & 165 & 127 & 100 \\
\hline
\end{tabular}

\begin{tabular}{|c|c|c|c|c|c|c|c|c|c|c|c|}
\hline CE & \multicolumn{2}{|c|}{ Impurity IV } & $\mathrm{CE}$ & \multicolumn{8}{|c|}{ Impurity V } \\
\hline 5 & 425 & & 5 & 441 & & & & & & & \\
\hline 10 & 425 & & 10 & 441 & & & & & & & \\
\hline 15 & 425 & & 15 & 441 & & & & & & & \\
\hline 20 & 425 & 191 & 20 & 441 & 385 & & & & 207 & & \\
\hline 25 & 425 & 191 & 25 & 441 & & & 288 & & 207 & & \\
\hline 30 & 425 & 191 & 30 & 441 & & 315 & 288 & 219 & 207 & & \\
\hline 35 & 425 & 191 & 35 & 441 & & 315 & 288 & 220 & 207 & & \\
\hline 40 & 425 & 191 & 40 & 441 & & 315 & 288 & & 207 & & \\
\hline 45 & & 191 & 45 & 441 & & 315 & 288 & & 207 & 137 & \\
\hline 50 & & 191 & 50 & 441 & & 315 & 288 & & 207 & 137 & 94 \\
\hline 60 & & 191 & 60 & 441 & & & 288 & & 207 & & 94 \\
\hline
\end{tabular}

Fig.5: The overlaid MSMS chromatogram of five impurities evaluated under 11 different collisions (55 data in 15 min) in ESI Negative mode

The positive mode $\mathrm{nMS}^{2}$ compiled fragments have given the similarity ion of $\mathrm{m} / \mathrm{z} 207$ ascertainging the presence of tetrazole ring in all the five impurities. The negative mode $\mathrm{nMS}^{2}$ compiled fragments have given the comparative differentiation 15 units leaves the prediction to the possibility of methyl/alkyl group variance in the irbesartan impurities. Thus the data analysis has enabled us to observe the presence or absence of a methyl group leading to the formation of impurity- 2 and impurity- 5 respectively. All the impurities except impurity-3 have their origin from the raw material spiroketones used in the synthesis of irbesartan. Impurity-3 is already reported as USP impurity-A [7].
To match the origin of the impurity, the synthetic root is screened carefully giving raise to the origin of the impurities are from the step II. Based on the MSMS spectral analysis both in positive and negative mode, the structure of the process related impurities with the molecular weight was shown in the schematic representation in fig. 6.

Thus the MSMS spectral analysis both in positive and negative mode has ascertained the possible correlations and necessitated variances with respect to irbesartan. This "nMS2" approach and the data correlation is highly important for the structural identification of any related impurities. The MSMS correlations provide the better understanding of the functional behavior. 


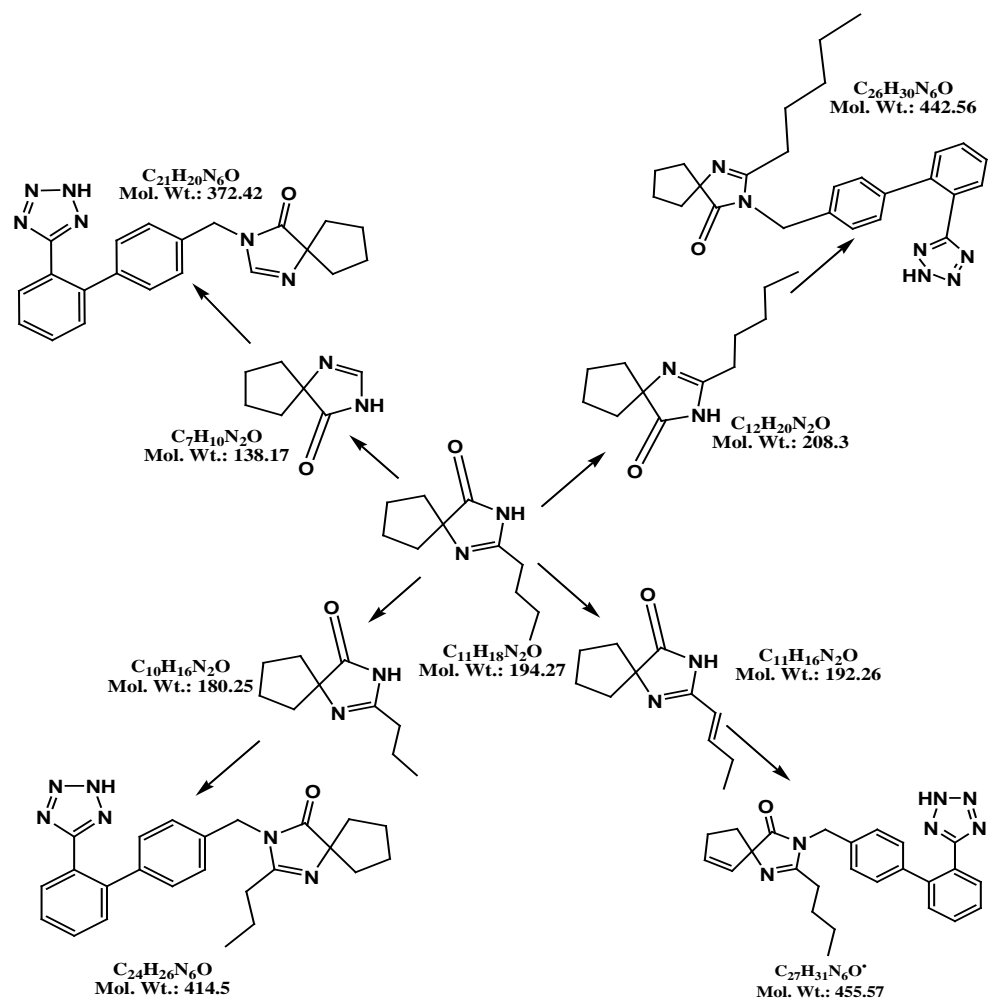

Fig. 6: The Schematic representation of Impurity origin in irbesartan

\section{CONCLUSION}

The MSMS approach of the "nMS" technique was evaluated in detail for the structural identification the trace level impurities of Irbesarton. On the successful evaluation of the impurities, it was observed that the impurities are a methylated, demethylated and alkenylated analogue of irbesartan. This approach is very fast and efficient as the mass identification is done after chromatographic resolution. The product ion formed was predominately from the parental ion thus the ion reliability is always higher and more meaningful data for interpretation. The mass segmental analysis with "nMS" approach is a technical advantage for the possible structural predictions of relative impurities in single analysis.

\section{ACKNOWLEDGMENT}

This research was supported by Indian Institute of Chromatography and Mass Spectrometry (IICMS) and SPICO BIOTECH. I express my deep sense of gratitude to Dr. Venkat Manohar, Director IICMS, Mr. Thyagarajan, Director SPINCO BIOTECH, Mr. Arvind Thyagarajan and Miss. Janani Thyagarajan for their support for this analytical research. I am thankful to our colleagues Mr. Mohan Kasi, Mr. Raman \& Dr. C. Govindarajan of IICMS team.

\section{AUTHORS CONTRIBUTIONS}

Mr. Saravanan Subramaniyan has made contributions to design the experiment and performed the same. Dr. Thaminum Ansari Abubacker has made a substantial contribution in drafting and reviewing the article content critically.

\section{CONFLICT OF INTERESTS}

All authors declare that there is no conflict of interest associated with this article

\section{REFERENCES}

1. RN Rao, V Nagaraju. An overview of the recent trends in the development of HPLC methods for the determination of impurities in drugs. J Pharm Biomed Anal 2003;3:335-77.

2. S Ahuja. Assuring quality of drugs by monitoring impurities. Adv Drug Delivery Rev 2007;59:3-11.
3. RJ Smith, ML Webb. Analysis of drug impurities. Blackwell Publishing, Oxford, UK; 2007.

4. J Zheng. Formulation and analytical development for low-dose oral drug products. John Wiley and Sons, Inc, Hoboken, New Jersey; 2009. p. 25-38.

5. Akshatha HS, Gurupadayya BM. Application of liquid chromatography coupled with mass spectrometry in the impurity profiling of drug substances and products. Asain J Pharm Clin Res 2018;5:30-7.

6. S Gorog. Identification and determination of impurities in drugs Elsevier, Amsterdam; 2000

7. SR Chemburkar, J Bauer. Dealing with the impact of ritonavir polymorphs on the late stages of bulk drug process development. Organic Process Res Development 2000;4:413-7.

8. KPR Chowdary, K Ravi Shankar, VVLSP Sowjanya. Optimization of irbesartan tablet formulation by 23 factorial design. Int J Curr Pharm Res 2015;7:39-42.

9. K Basak, HB Patel. Pharmaceutical impurities: a regulatory perspective for abbreviated new drug applications. Adv Drug Delivery Rev 2007;59:64-72.

10. D Annopkumar Anoop Kumar, Ganesh Darekar, Suma Ramagiri, Neha Bhasin, Manoj Pillai, Dev Kant Shandilya. Generic workflow using advanced analysis and data interpretation tools for identification of Irbesartan degradation products by liquid chromatography coupled to high-resolution mass spectrometry. Anal Chem Indian J 2015;15:352-63.

11. Somisetti Narender Rao, Devarasetty Sitaramaih, Challa Nageswar Rao, Peddi Srinivasa Rao, K Sudhakar Babu. Commercial scalable process for the preparation of irbesartan intermediate. Rasayan J Chem 2010;3:681-9.

12. Lars Peters, Roland Fr?hlich, Alan SF Boyd, Arno Kraft Tetrazole binding to amidine bases, Fifth International Electronic Conference on Synthetic Organic Chemistry; 2004.

13. Stanislav Radl, Jan Stach, Jaroslav Havlicek, Marcela Tkadlecova, Lukas Placek. Synthesis and identification of some impurities of Irbesartan. Acta Chim Slov 2009;56:559-65.

14. US. Pharmacopoeia USP30-NF25, Irbesartan (CAS: 138402-11-6); 2018. 